\title{
Amas de casa en el SIPA. Un análisis del plan de inclusión previsional como externalidad positiva al género
}

\author{
Housewives at the SIPA. An analysis of the Plan de Inclusión Previsional as a positive \\ externality to the gender
}

Tatiana Marisel Pizarro

Instituto de Investigaciones Socioeconómicas (IISE), Facultad de Ciencias Sociales de la

Universidad Nacional de San Juan, Argentina

tatianapizarro@conicet.gov.ar

Fecha de recepción: 17/12/2017, fechas de reenvíos: 20/04/2017 -16/06/2017, 10/08/2017 -23/08/2017, fecha de aprobación: 24/08/2017, fecha de publicacion:

07/09/2017

\begin{abstract}
Resumen: El presente artículo busca analizar el Plan de Inclusión Previsional como política cardinal de los gobiernos kirchneristas y cuyo fin fue anunciado como medida modificatoria del Sistema Integrado Previsional Argentino -SIPA- por parte del presidente Mauricio Macri.

Esta política resulta ser un objeto interesante de estudiar debido a que fue una moratoria para trabajadores autónomos que permitió que miles de amas de casa pudieran acceder a la jubilación. A lo mencionado, este artículo acopia nociones conceptuales de la "economía del cuidado", para expresar la vinculación existente entre el rol del cuidado en el sistema económico y las consideraciones reinantes por parte de las políticas públicas que refirieron una mayor inclusión, igualdad y protección durante los periodos 2004-2014.

En particular, es una observación del papel que ocupa la mujer y la visibilización de su rol como ama de casa en tanto ser merecedora de beneficios previsionales. Con este análisis se pretende exponer cómo esta política ha tenido como externalidad positiva, la ayuda a las mujeres que efectuaron labores informales o cuya invisibilización laboral las convirtió en trabajadoras vulnerables.
\end{abstract}

Palabras claves: jubilación; trabajo no remunerado; protección social; beneficios previsionales. 


\begin{abstract}
This paper aims to analyze the Pension Inclusion Plan (Plan de Inclusión Previsional) as a cardinal policy of the Kirchner governments, whose purpose was announced as a changing measure of the Argentinian Comprehensive Social Security System (Sistema Integrado Previsional Argentino - SIPA) by President Mauricio Macri.

This is an interesting policy to study since this disposition for self-employed workers allowed thousands of housewives to have access to a pension. This paper compiles conceptual notions of "Care Economy" to relate the role of care in the economic system and the main considerations in public policies that showed more inclusion, equality and protection during 2004 to 2014.

This paper particularly constitutes an observation of the women's role and a way to bring their role as housewives to light as workers deserving pension benefits. This analysis aims to explain how this policy has had a positive effect by helping women that conduct informal labor or whose work invisibilization has made them vulnerable workers.
\end{abstract}

Keywords: retirement; unpaid work; social protection; pension benefits.

\title{
Introducción
}

En la historia de la humanidad, el control sobre las mujeres por parte de los hombres hizo del patriarcado un modelo imperante, incluso en la sociedad actual. El patrón que se repite es el del hombre como jefe del hogar -quien ofrece su fuerza de trabajo a cambio de una remuneración con la que mantiene el grupo familiar-y la mujer a cargo de las tareas domésticas y de cuidado -quien también ofrece su fuerza de trabajo pero sin ningún rédito o beneficio social-.

Uno de los periodos de la historia más significativos estuvo dado con la industrialización, momento en que la unidad doméstica fue separada de la unidad de producción y se estableció una división sexual del trabajo más rígida. Ésta se convirtió, entonces, en una conformación social consolidada en el marco de sociedades capitalistas, en las que el espacio de la producción y la reproducción adquirieron límites más precisos (en relación con formas de producción precapitalistas donde las acciones de producción y reproducción se fundieron tanto en términos espaciales como temporales), provocando relaciones de género asimétricas, que subordinan la posición de las mujeres en la estructura económica y social.

Esto implica, entonces, discutir la concepción prevaleciente del trabajo exclusivamente como la actividad que produce bienes y servicios destinados al consumo o intercambio con vistas a satisfacer necesidades humanas, y darle relevancia a las actividades vinculadas con lo reproductivo del mundo del trabajo. La diferenciación entre lo "productivo" y lo "reproductivo" tiene que ver con que el primero se resume en un proceso de acumulación que utiliza las energías humanas como mercancía, mientras que el segundo aboca su tarea en reproducir esas energías como parte integrante de las personas (Picchio, 1994). 
En Argentina, en la última década, durante los periodos gubernamentales de Néstor Kirchner y Cristina Fernández de Kirchner se hizo un intento de revertir el hecho de haber soslayado la importancia de la labor del ama de casa a lo largo de su vida, dotándola de beneficios ya en su etapa de actor económicamente pasivo. Esto, a través de políticas de inclusión previsionales que permitieron a miles de ancianos y ancianas -entre ellas amas de casa- recibir una jubilación, pese a no haber realizado aportes para gozar de este beneficio.

El objetivo de este artículo es analizar al Plan de Inclusión Previsional como política que consideró a los trabajadores autónomos, dentro de los cuales se encuentran las amas de casa, como sujeto de derecho, al otorgarle valor económico a la labor que realizaron en el interior de los hogares. Por esto, el presente trabajo adopta como enfoque teórico la mirada de la economía del cuidado, tomando los principales conceptos de esta disciplina. De este modo, se pretende lograr un análisis más cabal de la medida analizada de acuerdo a los propósitos del estudio. Mediante el uso de la técnica de análisis documental y un miramiento a datos estadísticos oficiales sobre las implicancias económicas y políticas de esta medida, se pretende visibilizar la dimensión de género tácita en esta moratoria.

En la primera sección de este trabajo se hace una descripción sobre las construcciones culturales en torno al trabajo reproductivo. La segunda sección presenta a la mujer como beneficiaria indirecta de distintas medidas sociales y su mutación a beneficiaria directa de un haber jubilatorio. La tercera sección hace una descripción cronológica de la presencia del ama de casa en el SIPA. La cuarta sección expone las características del PIP y resultados en función a la autonomía económica de las mujeres ancianas beneficiarias a partir de datos de fuentes oficiales. Por último, en la quinta sección, se exponen las principales conclusiones del análisis realizado.

\section{El trabajo reproductivo no remunerado como formulador de fuerza de trabajo}

El escenario en el que se crean y reproducen las acciones que dan forma a la cohesión social es el seno familiar, y a su vez, es en éste en el que se reparten las cartas de poder entre las mujeres y los hombres. Es así que la mujer quedó principalmente a cargo de las tareas reproductivas dentro del hogar, en tanto el hombre pasó a desempeñarse en tareas productivas fuera de ese espacio, por las que empezó a recibir una remuneración.

De este modo las construcciones culturales transformaron esa rígida división sexual del trabajo en una especialización "natural" de las mujeres, en la que la familia y el hogar pasaron a ser considerados espacios de afecto y crianza, a cargo de ellas. Así, se racionalizaron dos creencias: la primera sostiene que el trabajo no remunerado en el hogar era trabajo de mujer y, la segunda, que en realidad no era trabajo, ya que no produce recursos materiales ni cuenta con prestigio social. 
Este cristal por el que se observó la realidad por siglos ha sufrido un quiebre con la feminización de los mercados laborales, que no sólo trajo aparejado el bienestar de los hogares y la disminución de la pobreza, sino que también marcó un cambio paradigmático en la forma de interpretar la multiplicidad de actores en el mercado laboral. Aun así, en la actualidad, es la mujer quien carga con la pretendida decisión de retirarse de la oferta de trabajo para dedicarse de manera principal o única al cuidado de los hijos e hijas. Ésta representa una acción en la que no sólo pesa el ideal "tradicional" de la división sexual del trabajo, sino también la evaluación acerca de la conveniencia (o no), de participar en un mercado laboral con oportunidades estrechas (o escasamente remuneradas), para mujeres pobres, frente a la escasez de servicios de cuidado gratuitos, así como los riesgos latentes que se asocian al cuidado por parte de personas desconocidas (Pereyra, 2012).

Esta realidad en la que la mujer se inserta en el mundo del trabajo remunerado y asume una enorme sobrecarga de trabajo doméstico por la desequilibrada distribución del trabajo reproductivo denota factores que hacen que la vida laboral y familiar sean totalmente inequitativas y contrastante con la realidad masculina. El trabajo reproductivo debe considerarse como un elemento necesario e imprescindible que influye en ámbitos que van más allá de lo meramente privado, ya que contribuye en forma directa en la persistencia del modo capitalista de producción -entiéndase que el sector capitalista requiere personal que ofrezca su fuerza de trabajo en toda su extensión-. Es decir, el trabajo doméstico proveería fuerza de trabajo al mercado para su venta.

\section{El cuidado en el Estado de bienestar}

Martínez Franzoni (2007), postula que los indicadores que determinan la demanda de trabajo no remunerado en los hogares "son tres: la cantidad de miembros del hogar por ama de casa, la cantidad de miembros del hogar que requieren cuidados y el tamaño total del hogar" ( $p$. 92). Lo que repercute en una gran cantidad de tiempo volcado por las mujeres en los trabajos domésticos, que suelen ser exclusivos de ellas, sin importar el régimen de bienestar o momento de la historia en el que se encuentre.

La mujer contemporánea, por ejemplo, negocia su trabajo no remunerado con el que sí lo es, desempeñándose exhaustamente en el ámbito doméstico y en una competencia constante en el mercado laboral. Gracias a las políticas de empleo (de desfamiliarización) y hacia la familia (familistas), ha sido posible mediar entre ambas realidades -a pesar de sus deficiencias: los mercados laborales, por lo general, resultan ser en gran medida informales y las políticas públicas deficientes- (Draibe y Riesco, 2006).

Es importante tener en cuenta que las políticas sociales son formuladas de acuerdo a ciertos conceptos de modelosfamiliares prevalecientes. Y los hogares interactúan con éstas para alcanzar 
determinados estándares de bienestar. Como señala Hintze (2004), "la unidad familiar genera o selecciona satisfactores para alcanzar sus fines reproductivos por medio de la combinación de las posibilidades a su alcance a través de un entramado de actividades que la relacionan con los demás agentes sociales" (p. 82). El problema, tal como lo señalan los estudios de las dimensiones de género de las políticas sociales, es que las instituciones sociales construidas en torno a estos dos organizadores básicos (el mercado laboral y las conformaciones familiares), fallan en reconocer las asimetrías, reproduciendo el papel subordinado de las mujeres .

Por esto, una ciudadanía basada en la igualdad de género supone una división sexual del trabajo, más igualitaria en el mercado de trabajo y respecto de los cuidados domésticos. Ya el fundamento de una ciudadanía basada en el reconocimiento de la diferencia supone que autonomía y libertad de elección respecto de la reproducción y de las actividades de cuidados constituyen otras bases de derechos específicos de la mujer -derechos reproductivos, derecho a la socialización de los cuidados domésticos- (Draibe y Riesco, 2006). Por esto, el abordaje del papel de las mujeres en el imaginario colectivo, merece una mirada que conjugue las necesidades de reconocimiento con las de redistribución (Fraser, 2003).

Respecto a esto, Fraser (2003), propone que las políticas de redistribución y las de reconocimiento no deben ser excluyentes entre sí, sino que por el contrario, deben armonizarse para alcanzar esa justicia social e inclusión. Es decir, es necesario "una política que pretenda combatir la exclusión social debe combinar una política de redistribución con una política de reconocimiento" (p. 56).

\section{Mujeres invisibles a las políticas públicas}

Debido a la peripecia inefable de acceder al mercado laboral formal, las mujeres se encuentran diariamente ante la dificultad de generar ingresos propios, lo que acarrea el riesgo de vivir situaciones de pobreza. En el caso de América Latina, se han impuesto los Programas de Transferencias Condicionadas de ingreso (PTC) como la principal forma de intervención de los gobiernos para atender a la población en situación de pobreza, mediante la garantía de cierto nivel básico (en general mínimo) de ingresos monetarios (Rodríguez Enríquez, 2011). A raíz de los PTC, el paternalismo estatal propagaba que la mejor forma de paliar las situaciones de pobreza era satisfacer las necesidades básicas de la población pobre a través de bienes y servicios, contraponiéndose al neoliberalismo que sostiene que la mejor forma de atender esta situación es a través de transferencias monetarias focalizadas y condicionadas (Rodríguez Enríquez, 2011).

Estos programas tienen como foco el bienestar de niños y niñas, por lo que las receptoras del beneficio son las mujeres de estos hogares, ya que se presume que son ellas las que velan por la prosperidad de sus hijos e hijas (Bertranou, 2011). Cabe destacar que el hecho de que estos programas tengan como destinatarias a las mujeres no los transforma en políticas con 
perspectiva de género. Pero, a pesar de esto, los PTC fomentan la igualdad de género, ya que la titularidad está dada a las mujeres -no como un derecho derivado-, permite el acceso a servicios de cuidado en contextos donde la estructura pública de este tipo de servicios es insuficiente y/o inadecuada; fomenta la inclusión de componentes que promueve la redistribución del trabajo remunerado y no remunerado, productivo y reproductivo, y dota de mecanismos de participación de la voz de las personas beneficiarias y de identificación y atención de las demandas específicas de las mujeres (Rodríguez Enríquez, 2011).

En esta línea, en Argentina durante la década kirchnerista para garantizar el "Derecho Familiar de Inclusión Social", se creó el Plan Jefas y Jefes de Hogar Desocupados (PJyJHD) dirigido a jefes de hogar desocupados con hijos e hijas menores de 18 años. También, se inició la ejecución del Programa Familias (Ingreso para el Desarrollo Humano), con el fin de promover el desarrollo, la salud y permanencia en el sistema educativo de los niños y evitar la exclusión social de familias en situación vulnerable. Bertranou (2011), explica que el IDH otorga subsidios -sujetos a contraprestaciones familiares en salud y escolaridad- a familias en situación de pobreza con hijos menores de 19 años y embarazadas que no reciban ayuda económica del Estado ni asignaciones familiares.

\section{Amas de casa en el sistema previsional argentino}

Ya en los años 90 hubo profundos cambios en la reforma del sistema previsional argentino (incremento en la edad y la cantidad de años de aportes exigidos para ser beneficiado previsionalmente) y en el mercado laboral -una gran parte de la población económicamente activa se quedó sin trabajo y sin protección social- (Observatorio para la Seguridad Social, 2011).

Con el gobierno de Carlos Menem se sanciona la Ley 24.241 y se crea el Sistema Integrado de Jubilaciones y Pensiones. Es con esta reforma previsional donde se estableció el régimen de capitalización individual por medio de las Administradoras de Fondos de Jubilaciones y Pensiones (AFJP). A partir de 1993, las amas de casa se incorporaron al régimen, pero esta incorporación resulta ser una "afiliación voluntaria autónoma especial", ya que sólo pueden ingresar régimen de capitalización del Sistema Integrado de Jubilaciones y Pensiones (Ley No24.828, 1997).

Dos años después, en 1995, con la Ley 24.476 , se le permitió regularizar la situación previsional a los/as trabajadores/as autónomos/as y tener así una forma de "pagar" los años no aportados. Entonces, tanto los/as trabajadores/as autónomos/as como las amas de casa, podían acceder en un futuro al beneficio de ser parte de un régimen previsional, siempre y cuando, éstos/as hayan cumplido con la edad estipulada y cancelado el monto total de los haberes requeridos. 
En el 2003, con la llegada al gobierno de Néstor Kirchnery teniendo en la formulación de políticas sociales como ejes conductores a la inclusión, solidaridad y universalidad, se estipularon distintas normas que permitieron la incorporación al sistema previsional de los/as más desventajados/as en la última década: autónomos/as, cuentapropistas y amas de casa.

Una de las políticas que cumplía con ese objetivo fue el Plan de Inclusión Previsional. Éste surge como medida de corto plazo para incorporar al ámbito de la seguridad social a aquellos adultos mayores que, castigados por los cambios registrados en el mercado de trabajo y en el sistema previsional en los años 90 , en particular referidos a la edad jubilatoria y los años de contribución necesarios para acceder a la prestación previsional, se encontraban en una situación de vulnerabilidad social al no contar con un haber jubilatorio.

El principal instrumento a través del cual se implementó el Plan de Inclusión previsional entre enero de 2005 y abril de 2007 fue el artículo 6 de la Ley 25.994, y el Decreto 1454/05, el cual reglamentó la Ley 24.476 y permitió que los/as autónomos/as con problemas de regularización de aportes, se puedan inscribir en un plan de facilidades hasta el 30 de abril de 2007 para acceder al beneficio previsional. De este modo, podían acceder al beneficio quienes teniendo la edad de retiro cumplida hasta el 31 de diciembre de 2004, no registraban aportes al sistema o los mismos eran insuficientes (estos aportes podían ser completados a través de la moratoria establecida en la Ley 25.865 y en las condiciones dadas hasta julio de 2004), y quienes cumpliendo la edad en cualquier momento, completasen los aportes anteriores al 30 de septiembre de 1993 (Calabria, Calero, D’Elía, Gaiada, Martínez y Rottenschweiler, 2012).

\section{Desigualdades de género en el sistema previsional}

Como anteriormente se postuló, es necesario subrayar que la desigualdad de género es una de las limitaciones más relevantes de los sistemas previsionales basados en el diseño bismarckiano. Arza (2013), plantea que en estos sistemas la unidad de protección es la familia, definida como un núcleo estable en el cual la mujer que se dedica al trabajo no remunerado del hogar (incluyendo cuidado de niños/as o ancianos/as) se encuentra protegida a través de su marido. El derecho a una pensión en caso de muerte es un ejemplo de esta cobertura "derivada" de la condición familiar. Uno de los problemas de este diseño es que responde cada vez menos a la realidad de la organización familiar actual. Por otro lado, a pesar de la tendencia al alza, aún hoy las mujeres siguen presentando tasas de participación en el mercado laboral menores que los hombres, por lo que a la hora de reclamar un beneficio jubilatorio contributivo se encuentran en desventaja: acumulan menos aportes y por lo tanto muchas de ellas no logran alcanzar el mínimo requerido para obtener un beneficio; y las que sí lo obtienen, reciben beneficios más bajos, sea por haber contribuido pocos años o porque sus aportes $y / o$ ingresos laborales fueron menores. 
Factores como la precarización de los mercados laborales, los cambios en las estructuras familiares y las restricciones fiscales durante los ' 80 y ' 90 llevaron a impulsar políticas de contención del gasto previsional, en lugar de políticas que permitieran ampliar la cobertura y los beneficios (Arza, 2013). Por esto, en la última década se vivió un proceso de contrarreforma que abarcó la lógica misma de funcionamiento del sistema (de un sistema mixto a un sistema de reparto), su administración (de mixta a estatal) y se crearon así programas que permitieron ampliar el número de beneficios y la cobertura, como es el caso de la política analizada.

\section{El plan de inclusión previsional y su externalidad positiva al género}

Con el Plan de Inclusión Previsional -PIP-, el Estado previó la inserción en el sistema jubilatorio de personas que incumplieran los requisitos previstos para el acceso a la prestación de vejez impuestos por el Sistema Integrado de Jubilaciones y Pensiones- aportes formales incompletos o ausencia de éstos, o bien aportes completos pero personas menores de 65 años-. Paulatinamente, el sistema previsional expandió su alcance mediante la implementación del Monotributo social y del Régimen de Regularización de Deudas de los/as trabajadores/as autónomos/as, que en su conjunto proponen saldar la deuda de contribuciones que tenían los aportantes previa a 1994. Cabe destacar que el Monotributo Social es una categoría tributaria permanente, creada con el objeto de facilitar y promover la incorporación a la economía formal de aquellas personas en situación de vulnerabilidad que han estado históricamente excluidas de los sistemas impositivos y de los circuitos económicos.

Aun así, ante esta situación, muchas mujeres se vieron en la obligación de declarar alguna actividad "productiva" a fin de acceder a este beneficio, y no presentarse a sí mismas como "amas de casa" al no considerarse éste un trabajo propiamente dicho, excluyéndola así del derecho que el trabajador en su etapa de retiro tiene: la jubilación. El abordaje de esta cuestión se vincula la necesidad de diseñar sistemas de pensiones que, o bien reconozcan el costo de continuidad y calidad de los empleos que soportan quienes sostienen la carga no remunerada del trabajo, o bien prevean que una proporción importante de la pensión futura no dependa del vínculo formal en el mercado laboral (CEPAL, 2009).

El trabajo de las mujeres constituye un todo inseparable y el proceso de reproducción social está interrelacionado con otros procesos socioeconómicos por lo que si se analiza el trabajo reproductivo aislado del trabajo productivo y del proceso de reproducción social, se contribuye a ocultar la importancia, la complejidad y dimensión del trabajo doméstico. Al relacionar el trabajo reproductivo con su papel social, el trabajo femenino es entonces un tema para todo el sistema, no se trata ya de un problema específico de las mujeres (Lagarde, 1990).

En la primera moratoria previsional en 2004, de los 2,7 millones de personas que pudieron acceder al beneficio (con un promedio de ocho o diez años de aportes), el $73 \%$ fueron mujeres. Diez años 
después, durante la segunda ola de la moratoria previsional, del total de beneficiarios/as un $86 \%$ fueron mujeres y el $14 \%$ varones. La diferencia es notoria: ocho de cada diez beneficiarias de esta medida son mujeres (Peker, 2016). Sin tenerlo como su objetivo primordial, esta política ha tenido como externalidad positiva la ayuda a las mujeres que efectuaron labores informales o cuya invisibilización laboral las convirtió en trabajadoras vulnerables. Al analizar los resultados de la moratoria, Anses (2010) sostiene que:

Es un hecho estilizado que la mayoría de los regímenes de pensiones contributivos no brindan cobertura frente riesgos específicos asociados a la maternidad y a las responsabilidades familiares históricamente asignadas a la mujer, tales como el cuidado de los niños, ancianos y enfermos. Asimismo, las trayectorias interrumpidas de empleo y los salarios más bajos en general llevan también a que cada vez menos mujeres participen en un régimen de pensiones contributivo durante su trayectoria laboral y reciban beneficios durante la edad de retiro (AISS, 2002).... En este sentido, el Plan de Inclusión Previsional ha tenido un papel activo en favor de la igualdad de género en los regímenes de pensiones (p. 26).

Con el nuevo gobierno de corte neoliberal del presidente electo Mauricio Macri, se anunció el fin de estas moratorias. De este modo, se concluyó con una solución transitoria a las consecuencias de políticas del mismo modelo tomadas en los años 90 . Este escenario político, como se mencionó antes, condujo a que miles de ancianos y ancianas no tuvieran los años de aportes previsionales requeridos para acceder a una prestación, lo que los llevó a una clara situación de vulnerabilidad social. Ante esta realidad, cabe aclarar que las mujeres siempre han sufrido informalidad laboral, que las obliga a aceptar trabajos con evasión de aportes patronales, dejándolas sin jubilación ni obra social, entre otros beneficios.

Cabe destacar que esta moratoria fue una medida efectiva e inmediata al cumplir con una cobertura previsional casi total de la franja etaria de los 60 a 64 años, reconociendo el derecho universal de la ancianidad a acceder a protecciones previsionales. Contraponiendo esta realidad, deviene la decisión del gobierno macrista de no renovar las moratorias previsionales, ya que propone una reformulación del sistema jubilatorio que recuerda al vigente en la década de los 90 -consistente en la transformación del actual modelo de reparto por una pensión universal de la vejez que para acceder prolonga la edad jubilatoria y corresponde a un $80 \%$ del monto total de una jubilación considerada mínima-.

El escenario es similar, la vigente recesión económica al igual que la reciente ola de despidos que asciende a 143.423 según el informe de CEPA (2016)-, son determinantes en la reducción de la generación de empleo, lo que recae en un desfinanciamiento de las cajas de seguridad social y en la interrupción del sistema de solidaridad intergeneracional propio del sistema de reparto (Letcher y Strada, 2016). 
El Plan de Inclusión Previsional no sólo fue una medida para universalizar las prestaciones previsionales a ancianos y ancianas con vulnerabilidad social, sino que fue el reconocimiento implícito que las amas de casa ancianas alcanzaron como trabajadoras visibilizadas.

\section{Reflexiones finales}

Con este trabajo se pretendió analizar mediante una perspectiva de género las transformaciones en el Sistema Integrado Previsional Argentino suscitadas con la inserción del ama de casa como beneficiaria directa del Plan de Inclusión Previsional.

El predominio femenino de la titularidad de las moratorias da cuenta de cómo esta medida resultó ser un divisadero de la realidad de aquellas mujeres mayores que tuvieron una trayectoria laboral intermitente, informal o realizaron trabajos domésticos no remunerados a tiempo completo, quedándose sin seguridad social en la ancianidad.

Es preciso que la valoración del trabajo de cuidado no remunerado incida en políticas públicas que reconozcan y velen por una mayor equidad y autonomía para las mujeres, esto contribuye al bienestar y desarrollo de capacidades humanas y, asimismo, al crecimiento económico, debido a que agrupa la mayor cantidad de horas de trabajo que equivaldría más de la mitad del PBI (Gómez Gómez, 2008). En otras palabras, las mujeres no sólo realizan la mayor parte de este tipo de labores, sino que al sumar sus jornadas de trabajo remunerado y no remunerado, ellas desempeñan jornadas más extensas que los varones.

Si bien los Estados tienen el deber de proteger y promover los derechos de toda la ciudadanía, principalmente los de los oprimidos o desventajados socialmente -en ambas categorías encaja la mujer-, comprender y desnaturalizar la labor doméstica como propiamente femenina y realmente visualizarla como tal, es una tarea ecuménica. Este tipo de cambio transformativo en el sistema previsional permite desaprender y liberarse de aquellas mentalidades, relaciones, identidades que entorpezcan las creaciones de nuevas realidades más justas y equitativas en términos políticos, sociales y económicos.

Con estas moratorias previsionales se puso en foco la concepción de las tareas domésticas no remuneradas y el valor social de éstas como trabajo. Si desde sus inicios, el PIP hubiese tenido entre sus objetivos principales la inclusión del ama de casa al sistema previsional, éste hubiese sido un modelo ejemplificador de la importancia de articular políticas sociales, económicas y culturales con una perspectiva de género.

La realidad es que su implementación tuvo implicancias de género por una externalidad positiva de su objetivo original -extender la cobertura previsional a personas sin registros contributivos suficientes-. Es decir, sin pretenderlo, esta política desafió a la desigualdad de género, los 
imaginarios y la naturaleza de la actividad como persona trabajadora activa, reconociendo el valor que tiene la labor del ama de casa al suministrarles beneficios monetarios básicos.

En esta línea, es necesario enfatizar que a pesar que el PIP resultó ser para las amas de casa un vehículo para alcanzar un nivel de autonomía económica desconocida y una concepción de sujetas merecedoras de este derecho, esto no implicó cambios en la división sexual del trabajo ya que la medida no manifestó un reconocimiento explícito del trabajo reproductivo. En otras palabras, estas moratorias compensaron una visible desigualdad existente en la inserción de trabajadores/as autónomos/as con problemas para acceder al sistema previsional pero no transformaron la invisibilidad otorgada al trabajo realizado en seno privado del hogar, por lo que las jubilaciones otorgadas a las amas de casa resultaron ser un beneficio no previsto para ellas.

Tras décadas de gobiernos populares y neoliberales, la deuda sigue siendo la misma: una nómina de políticas que continúan invisibilizando a las trabajadoras que desempeñan tareas no remuneradas en el ámbito privado del hogar, siendo las que proveen fuerza de trabajo al mercado para su venta.

\section{Referencias bibliográficas}

Anses (2010). Análisis de la Cobertura Previsional del SIPA: Protección, Inclusión e lgualdad. Buenos Aires, Anses. Recuperado de http://observatorio.anses.gob.ar/archivos/documentos/ An\%C3\%A1 lisis\%20de\%20la\%20Cobertura\%20del\%20SIPA.pdf

Arza, C. (2013). La política previsional y la prevención de la pobreza: elementos salientes de la trayectoria latinoamericana reciente. Revista Voces en el Fénix. №23, 102-109. Buenos Aires. Recuperado de http://www.youblisher.com/p/616267-Voces-en-el-Fenix-N-23-Nosotroslos-pobres/

Bertranou, F. (2011) Encrucijadas en la seguridad social argentina: reformas, cobertura y desafíos para el sistema de pensiones. Buenos Aires: CEPAL y Oficina Internacional del Trabajo. Caps. 3 y $6,71-92$ y $131-149$.

Calabria, A., Calero, A., D’Elía, V., Gaiada, J., Martínez, J. y Rottenschweiler, S. (2012). Análisis del Plan de Inclusión Previsional: protección, inclusión e igualdad, Revista de Economía Pública, Social y Cooperativa. N74, 285-311. CIRIEC-España, Recuperado de http://www.redalyc.org/ pdf/174/17423124011.pdf

CEPA (2016) Informe de coyuntura económica. Centro de Economía Política. Argentina. Abril de 2016. Recuperado de http://www.centrocepa.com.ar/coyuntura-abril-2016.pdf 
CEPAL (2009). Género, trabajo remunerado y no remunerado: eslabones en la discriminación y la desigualdady Políticas Públicas y crisis de cuidado en América Latina: alternativas e iniciativas. En CEPAL (2009) Panorama Social en América Latina 2009 (pp. 173-197), Santiago de Chile, Chile: CEPAL.

Draibe, S. y Riesco, M. (2006). Estado de Bienestar, desarrollo económico y ciudadanía: algunas lecciones de la literatura contemporánea. Serie Estudios y perspectivas 55. México: CEPAL.

Fraser, N. (2003). Redistribución, reconocimiento y exclusión social. En: Inclusión social y nuevas ciudadanías. Bogotá, Colombia: Departamento Administrativo de Bienestar Social. DABS \& Pontificia Universidad Javeriana.

Gómez, G. (2008) La valoración del trabajo no remunerado: una estrategia clave para la política de igualdad de género, En: CEPAL (2008). La Economía Invisible y las desigualdades de género. La importancia de medir y valorar el trabajo no remunerado. Washington, D.C. OPS, CEPAL, Consejo Superior de Investigaciones Científicas.

Hintze, S. (2004). Capital social y estrategias de supervivencia. Reflexiones sobre el capital social de los pobres. Recuperado de http://www.flacsoandes.edu.ec/agora/capital-social-y-estrategiasde-supervivencia-reflexiones-sobre-el-capital-social-de-los

Lagarde, M. (1990). Cautiverio de las mujeres: madres, esposas, monjas, putas, presas y locas. México: UNAM.

Letcher, H. y Strada, J. (24 de abril de 2016) Se jubiló la moratoria. Suplemento Cash. Página 12. Recuperado de http://www.pagina12.com.ar

Lewis, J. (1993), Women and Social Policies in Europe: Work, Family and the State. Aldershot, Inglaterra: Edward Elgar.

Ley N²4.828, 26 de junio de 1997. Sistema integrado de jubilaciones y pensiones Amas de Casa. Adóptense medidas para su ingreso al citado Sistema. Buenos Aires, Argentina. Recuperado de: http://servicios.infoleg.gob.ar/infolegInternet/anexos/40000-44999/44189/norma.htm

Martínez Franzoni, J. (2007) Regímenes del Bienestar en América Latina. Madrid: Cealci Fundación Carolina. DT 11, Cap. 1 y 3.

Observatorio para la Seguridad Social (2011). Análisis de la cobertura previsional del SIPA: protección, inclusión e igualdad. Recuperado de http://observatorio.anses.gob.ar/archivos/ documentos/An\%C3\%A1lisis\%20de\%20la\%20Cobertura\%20del\%20SIPA.pdf 
OIT- PNUD (2009) Trabajo y familia: Hacia nuevas formas de conciliación con corresponsabilidad social. Santiago: OIT-PNUD.

Pereyra, F. (2012). La regulación laboral de las trabajadoras domésticas en Argentina: situación actual y perspectivas. En: Esquivel, V; Faur, Ey Jelín, E. (2012) Las lógicas del cuidado infantil. Entre las familias, el Estado y el mercado. Buenos Aires: IDES 2012.

Peker, L. (18 de marzo de 2016). Sin Resguardo. Suplemento Las 12. Página 12. Recuperado de http:// www.pagina12.com.ar

Picchio, A. (1994) El trabajo de reproducción, tema central en el análisis del mercado laboral. En Borderías, C., C. Carrasco y C. Alemany (1994) Las mujeres y el trabajo. Recuperado en http://www.ccee.edu.uy/ensenian/catgenyeco/Materiales/2011-08-10\%20M3\%20\%20 Picchio(1994)-ElTrabajoDeReproduccion.pdf

Rodríguez Enríquez, C. (2011). Programas de Transferencias Condicionadas de Ingreso e Igualdad de Género. ¿Por dónde anda América Latina? Serie Mujer y desarrollo 109. Santiago, Chile: Cepal. 